\title{
BITS, BYTES E QUALIDADE DE VIDA: uma breve reflexão sobre design, sustentabilidade e a era da informação.
}

\author{
Michelle Cotrim \\ UEMG \\ michellecotrim@hotmail.com \\ Prof. Dr. Lia Krucken \\ UEMG \\ lia.krucken@pq.cnpq.br
}

Resumo: A sociedade contemporânea tem experimentado uma espécie de miniaturização e desmaterialização das 'coisas' - argumenta Flusser (2007). Este pensamento é corroborado por Friedman (2005), que aponta que a comunicação cada dia mais ocorre por meio de bits que navegam pelo ciberespaço. Mas as aventuras cibernéticas não se condensaram no âmbito comunicacional, expandiram-se e, de acordo com Carroll e Romano (2011), tem ocupado uma parte crescente de nossa existência na forma de informações digitais. Com base nestas proposições o presente artigo procura, por meio de revisão bibliográfica e análise semiótica, apresentar uma reflexão sobre as relações entre sustentabilidade (qualidade de vida e bem estar social) frente as mídias digitais, utilizando como metáfora para a era digital o romance de Lewis Carroll (1920), Alice no País das Maravilhas.

Palavras-chave: Era Digital , Alice no País das Maravilhas, Estilo de Vida Sustentável, Cyber-Sustentabilidade.

Resumo: Contemporary society has experienced a kind of miniaturization and dematerialization of 'things' - argues Flusser (2007). This hypothesis is corroborated by Friedman (2005), who points out that communication increasingly occurs through bits navigating through cyberspace. But cyber adventures had not been condensed in the communicational context, expanded and, according to Carroll and Romano (2011), has occupied an increasing part of our existence as digital information. Based on these propositions this article seeks, through literature review and semiotic analysis, present a reflection on the relationship between quality of life and social well-being across digital media, using as a metaphor for the digital age the novel by Lewis Carroll (1920), Alice in Wonderland.

Keywords: Digital Age, Alice in Wonderland, Sustainable Lifestyle, CyberSustainability 


\section{INTRODUÇÃO: DESMATERIALIZAÇÃO X ESTILO DE VIDA SUSTENTÁVEL}

Alguns autores entendem que o processo de desmaterialização (com destaque para Manzini e Vezzoli,2002, que abordam esse tema no âmbito do design) pode ser entendido como um dos caminhos a ser buscado rumo a uma sociedade sustentável. Mas será que ao virtualizarmos a vida estamos caminhando nessa direção?

Ao propormos uma discussão entre design, sustentabilidade e digitalização/virtualização se faz necessário retomar brevemente alguns conceitos. 0 primeiro é o entendimento quanto a sustentabilidade, compreendido neste artigo por viés holístico. Com base em Ehrenfeld (2008), autor que discuti a relação entre sustentabilidade e design, "sustainability is first a human problem and then a environmental problem. If we fail to adress the unsustainability of the modern human being, we wil not be able to come with others aspects of sustainability (EHRENFELD, 2008, p.97) . E de acordo com Hoffman (2013), (co-autor com Ehrenfeld na obra Flourishing: a frank conversation about sustainability):

sustainability is not fixed in state to be achivied but a constant reaching for what it truly means to be a human being living in a interconnected and complex world. It is a desirable future; one built not just on technological and material development but also on cultural, personal and spiritual growth. (EHRENFELD, 2013, p.07)

O segundo aspecto diz respeito a conceituação de sociedade sustentável, o qual foi baseado em Jurin (2012), que pontua que " uma sociedade sustentável envolve o sistema sociocultural que mantém as pessoas em qualidade de vida" (JURIN, 2012,p.02). Nesse sentido, Herculano (2000) propõe um questionamento:

haverá fatos novos na vida social contemporânea que expliquem o surgimento da preocupação, no plano teórico-epistemológico, com a qualidade de vida e com sua proposta como sendo uma alteração paradigmática? O despontar e expansão de novos movimentos sociais ambientalistas, de defesa dos direitos civis e humanos, contraculturais, alternativos, étnicos, de gênero, etc. - são vistos como evidências disso." (HERCULANO,2000, p.29)

O terceiro ponto a ser considerado trata da digitalização/virtualização, para o qual nos apoiamos em Vilches (2003). Segundo o este autor "a migração digital diz respeito, em primeiro lugar, a sujeitos interconectados que chegam à fronteira da comunicação e do real". O autor complementa que "essa nova fronteira, que alguns chamam de ciberespaço, é um novo espaço de pensamento e de experiências humanas, formado pela coabitação de antigos meios e novas formas de hiperrealidade." (VILCHES,2003,p.17)

Baseado nesses questionamentos e apontamentos, o objetivo deste artigo é apresentar uma reflexão sobre relações entre qualidade de vida e bem estar social frente as mídias digitais, utilizando como metáfora para a era digital o romance de Lewis Carroll (1920), Alice no País das Maravilhas. O artigo está organizado em 4 partes. Primeiramente apresenta-se a constante e crescente digitalização da vida contemporânea. Na sequência é discutida a miniaturização e compactação de produtos, serviços e individualidades. A seguir é apresentada um breve paralelo entre a era da informação e a busca por um estilo de vida sustentável. Por fim, são apresentadas algumas considerações sobre o papel do design na articulação e mediação entre os pontos acima discutidos. 


\section{NA TOCA DO COELHO: A DIGITALIZAÇÃO DA VIDA}

Alice estava entediada colhendo margaridas no campo quando viu um Coelho que reclamava estar atrasado, fato que apesar de absurdo, não the desperta estranheza, "mas no preciso momento em que o Coelho tirou um relógio do bolso do colete, olhou para ele e começou a correr mais depressa. Alice pôs-se em pé de um pulo (...) . Ardendo de curiosidade, começou a correr pelo campo, atrás dele (...)". (Carroll,1920,p.06)

Assim como Alice, podemos dizer que nos encontrávamos entediados pela ordem e solidez da modernidade e quando avistamos a oportunidade de nos desvencilharmos da rigidez e do aprisionamento do tempo, nos dispusemos a segui-la impetuosamente, cada vez mais, nos convertemos em bit e bytes e passamos a habitar a nuvem, um espaço no qual o tempo não é real. Rushkoff (2010) argumenta que como as "atividades online não ocorrem em um tempo real, acabamos por ter todo o tempo do mundo." (RUSHKOFF, 2010,p.24)

O computador passou a ser o centro de nossas atenções, Lévy aponta que o mesmo "não é apenas uma ferramenta a mais para a produção de textos, sons e imagens, é antes de mais nada um operador de virtualização da informação" (Lévy,1980,p.55). Podemos dizer que na sociedade contemporânea muito de nossas posses se encontram na esfera digital. Friedman (2005) afirma que "estamos entrando num mundo em que um volume cada vez maior de comunicação se dá sob a forma de bits que viajam pelo ciberespaço e são armazenados em servidores espalhados por todo o mundo" (FRIEDMAN, 2005, p.251).

Devido a complexidade e a integração da tecnologia no cotidiano contemporâneo "nós hoje vivemos, como seres tecnológicos, em uma tecnosfera que foi incorporada a natural - não é mais possível localizar a linha divisória entre estas" (FRY,2011,p.55). Portanto entendemos que vivemos em um estado híbrido e indivisível, parte natural e parte tecnológico. Carroll e Romano (2011) compartilham deste pensamento e apontam que "com muitas de nossas posses na forma digital e novos ativos constantemente sendo criados, uma parte significante e em crescimento de nosso estado é hoje digital" (CARROLL et. al., 2011, p. 41).

Da mesma forma que Alice entra na toca do Coelho sem saber como iria sair, nós nos rendemos aos encantos tecnológicos da era digital sem nem ao menos considerarmos a direção pela qual optamos. Enquanto perseguíamos o Coelho, caímos em um poço e após o susto inicial, como Alice, passamos a olhar ao redor e apesar de não conseguirmos ver o fim, ou o fundo, percebemos todo conteúdo ao qual temos acesso. Tapias (2006) argumenta que "embora seja verdade que estamos muito perdidos, (...) observamos que, (...) se tornou impossível para nós pensar em retorno. (TAPIAS,2006,p.08)

Um ponto interessante é que até a pouco tempo para nos conectarmos era necessário um computador, uma linha telefônica (fixa) e discarmos para um modem servidor. Hoje temos tudo isso na palma das mãos através dos smartphones. Chatfield (2012) aponta que

estamos a passar progressivamente da informática pessoal para aquilo que podemos chamar de informática íntima. (...) Para a geração dos chamados nativos digitais, o celular é muitas vezes a primeira coisa em que se toca quando acorda e a última em se mexe antes de ir para a cama à noite" (CHATFIELD,2012) 
A facilidade de conexão à rede apresentada nos dias atuais, proporciona que em questão de segundos sejamos dragados para um universo paralelo por muitas vezes alheios ao que nos rodeia. Podemos nos perder em meios as informações e imagens: mapas, redes sociais, emails, livros e apesar de estarmos cercados por seres humanos nos contentamos em compartilhar e curtir em uma esfera virtual; ao mesmo tempo que estamos presentes, pelo menos em corpo, nossas mentes estão dispersas.

\section{BEBA-ME: AS INFINITAS POSSIBILIDADES DA NUVEM}

Seguindo o Coelho, Alice encontra um corredor, cercado por portas, as quais testa e percebe que estão trancadas, ao correr os olhos uma segunda vez, encontra uma mesa (que não parecia estar ali na primeira vez) e em cima desta uma chave dourada, que devido ao seu pequeno tamanho não poderia pertencer a nenhuma das portas testadas.

De repente Alice avista uma pequena porta escondida detrás de uma cortina. "Alice abriu a porta, e descobriu que esta dava acesso a uma passagem (...) ela se ajoelhou e olhou ao longo da passagem para o jardim mais bonito que você já viu." (CARROLL, 1920,p.09) (figura 1)

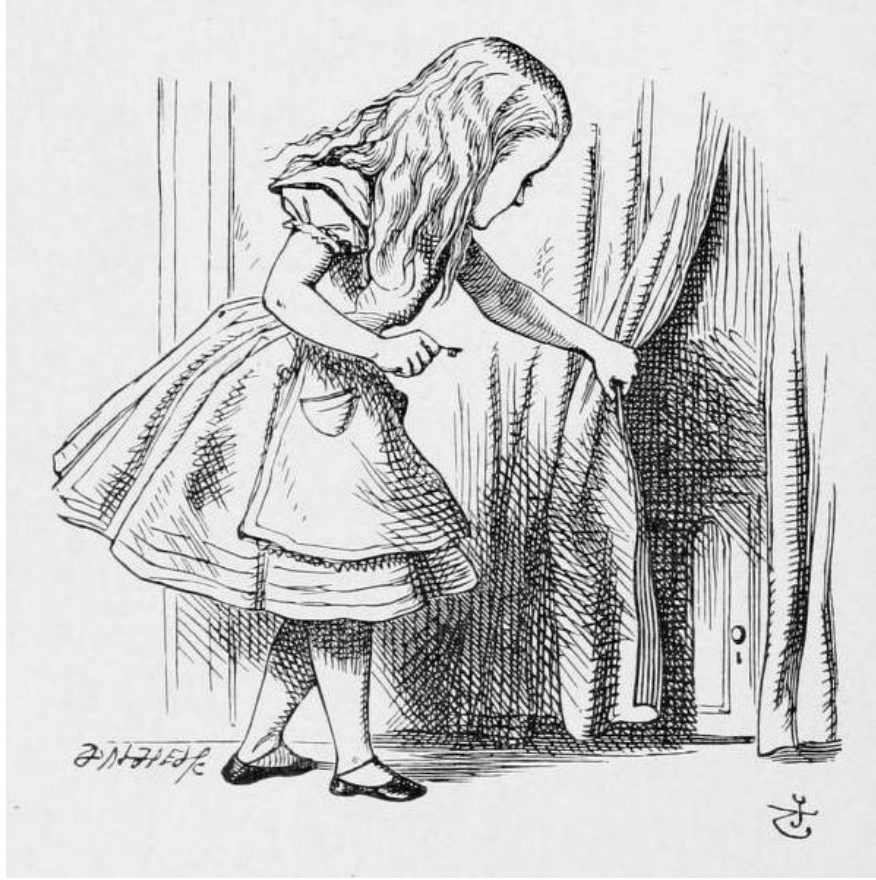

Figura 1- Ilustração Alice . Fonte: EBOOKS..., 2014.

Encantada com as possibilidades, Alice deseja que pudesse encolher, como um telescópio, para que tivesse acesso ao jardim inacreditável, e como tantas coisas extraordinárias já haviam acontecido naquele dia, "Alice começou a pensar que muita poucas coisas eram realmente impossíveis" (CARROLL, 1920,p.09).

Assim como Alice passou a acreditar no fantástico, Chatfield argumenta que, graças a internet, "vivemos numa era de milagres tão banais, que pode tornar-se difícil não os encarar como parte da textura cotidiana" (CHATFIELD, 2012), e como Alice desejou diminuir para ter acesso ao jardim, nós seres tecno-naturais, ao vislumbrar as infinitas possibilidades que a nuvem nos oferece, também desejamos que pudéssemos 'diminuir', nos miniaturizar, para então estarmos adequados a fluidez da rede. 
Flusser (2007) aponta que "o entorno está se tornando progressivamente mais impalpável, mais nebuloso, mais fantasmagórico, e aquele que nele quiser se orientar terá de partir desse caráter espectral que Ihe é próprio" (FLUSSERr, 2007,p.55). 0 autor descreve que "as coisas ao nosso redor estão encolhendo, em uma espécie de miniaturização" (FLUSSER, 2007,p.62). Desta forma podemos expandir estes conceitos e aplicá-los ao ser humano, uma vez que passamos a nos digitalizar, converter em zeros e uns parte de nossa existência. Carroll e Romano (2013) corroboram com esta afirmação e argumentam que vivemos uma vida digital em ascensão, na qual a produção de dados cresce a cada dia, e os autores pontuam que estes são mais do que meros dados computacionais, passam a se configurar como um conjunto de artefatos passíveis de contar a história de nossas vidas.

Alice, na busca por uma alternativa que a encolha encontra um garrafa com tal ordem: "BEBA-ME"; uma sentença imperativa disfarçada de convite. Alice deduz que como não tem nenhum aviso de veneno o líquido não é perigoso e sem refletir nas consequências toma um gole; percebe que começa a diminuir e "sua face se ilumina ao pensar que agora ela tem o tamanho certo para passar pela porta."(CARROLL, 1920,p. 11)

Não obstantes a Alice diariamente somos 'convidados', por ordens imperativas, a acessar, curtir, compartilhar, baixar, e como a personagem, antes de mergulharmos de cabeça verificamos rapidamente se não há perigo, se não é veneno... e quando acreditamos que é 'seguro' nos atrevemos a experimentar sem medir as consequências.

\section{PAÍS DAS MARAVILHAS? A ERA DA INFORMAÇÃO E CYBER-SUSTENTABILIDADE}

Ao nos conectarmos a rede aos poucos deixamos de nos conectar pessoalmente. Rushkoff (2010) aponta que “(...) a rede é melhor ao criar simulações e aproximações de interação humana a uma grande distância do que fomentar interações entre pessoas em um mesmo lugar." (RUSHKOFF, 2010,p.38). Através dos smartphones e aplicativos como What'sapp, Facebook, Instagram priorizamos nos comunicar com pessoas ao redor do globo, mesmo que estejamos cercados fisicamente por outros indivíduos.

O mesmo autor sugere "que associamos as telas de nossos computadores e nossas contas de email com as mais profundas experiências de comunidade e conexão, e tomamos seções de comentários de blogs como conversas extremamente significantes." (RUSHKOFF, 2010,p.29).

Passamos a depender da rede para nossas interações, mas lembrando que estamos sempre atrasados, Rushkoff articula que ao invés de nos tornarmos mais eficientes e capazes, nos tornamos frágeis e exaustos. Verificamos o aparelho de celular a cada segundo, acessamos nossas contas de email e perfis em redes sociais incessantemente. A fatiga e o estresse nos leva a desenvolvermos síndromes, como por exemplo a síndrome da vibração fantasma (phantom vibration syndrome), enquanto, de acordo com Vilches, a contínua dedicação a jogos em redes e chats podem levar à síndrome da personalidade múltipla.

Ainda em Vilches temos que "os sujeitos que entram na rede desvinculam-se da sociedade" (VILCHES, 2003,p. 22) e que "a falta de experiências sociais fora dos meios e a carência de atividade que permitam um desenvolvimento harmônico do corpo e 
das emoções poderiam estar formando gerações de difícil adaptação ao mundo real." (VILCHES, 2003,p.38)

Desta forma, destaca-se o pensamento de Morin (2003) que sugere que "o enfraquecimento de uma percepção global leva ao enfraquecimento do senso de responsabilidade (...) bem como ao enfraquecimento da solidariedade" (MORIN, 2003,p.18). Nesse sentido é oportuno citar Flint (2001) a respeito do desenvolvimento sustentável:

O caminho para um desenvolvimento sustentável só pode ser construído
através de uma mudança na psique humana em prol de uma
conscientização mais profunda. Qualidades tais como compaixão, paciência,
confiabilidade, coragem, humildade, cooperação, e disposição de se
sacrificar pelo bem comum, formam a base essencial, mesmo que invisível,
para a sociedade humana. (FLINT, 2001,p. 139)

As tensões entre tecnologia e sustentabilidade vêm sendo discutidas por diversos autores, dentre estes pode-se destacar Jurin (2012) e Knowles et. al. (2013). Jurin (2012) aborda a temática ao propor que "um estilo de vida sustentável não pode incluir orgulho excessivo e fé no estilo de vida tecnologicamente avançado sem reconhecer que esta mesma tecnologia pode nos guiar a eventual ruína."(JURIN, 2012,p.03)

Knowles et. al. (2013) tem introduzido o termo cyber-sustentabilidade, de acordo com os autores "we present the concept of cyber-sustainability as a necessary dimension of any sustainability discussion in our higly digitized world." (KNOWLES, 2013, p.488). Segundo os mesmos "cyber-sustainability, therefore, must deal with considerations about environmental, social and spiritual issues, while also acknowledging the economic dimension currently functioning as a barrier to change." (KNOWLES apud WALKER, 2013, p.488)

Com bases nessas considerações propomos um momento de reflexão e a avaliação em como o design pode fomentar e promover um estilo de vida sustentável , levando em consideração a qualidade de vida e a era digital.

\section{CONSELHOS DE UMA LAGARTA: DESIGN, DIGITALIZAÇÃO E SUSTENTABILIDADE}

"Who are you? Said the Caterpillar. (...) Alice replied, rather shyly, "I - I hardly know, Sir, just at present - at least I know who I was when I got up this morning, but I think I must have been changed several times since then." (CARROLL,1920,p.53) Neste trecho percebemos a indagação da Lagarta sobre a identidade de Alice, e confusa perante a tantas mudanças em um curto espaço de tempo, Alice não se reconhece mais. Da mesma forma ao encolher, esticar, expandir, miniaturizar e digitalizar, mergulhamos em bits e bytes, aos poucos nos desprendemos do nosso potencial de humanidade, ficamos confusos em uma deriva cibernética e insustentável.

Assim como a Lagarta questiona Alice, Hoffman propõe que

If we are going to address sustainability fully and meaningfully, (...) we must make fundamental shifts in the way we think an the way we organize our society. (...) these are "paradigm shifts" (in the words of the philosopher Tomas Kunh), changes in the way we think about ourselves, each other, and the world around us. In short sustainability takes a movement to reexamine who we are, why we are here, and how we are connected to every thing around us. [...] the transformation must be based on recognition that the old way of thinking no longer works; otherwise it will not solve the problem that it has created. (EHRENFELD, 2013, p.04) 
Da mesma maneira que foi necessário uma mudança de perspectiva para Alice pois, o biscoito COMA-ME e o líquido BEBA-ME não representavam mais a solução para seu problema de constante alteração de tamanho, foi necessário a intervenção da Lagarta para que Alice se lembrasse de quem era e voltasse a se reconhecer.

Walker (2013) pontua que "perhaps the most important role for design today is to explore ways of reuniting our material world with world of meaning - with ethics, inner growth, and spiritual wellbeing"(WALKER, 2013,p.446), talvez este seja o papel do designer, intervir assim como a Lagarta e nos lembrar do nosso potencial de humanidade.

\section{CONSIDERAÇÕES FINAIS:}

Dada a natureza do artigo se faz inevitável comentar que sem as ferramentas que o meio digital oferece, sejam elas: o scholar.google (que permite em segundos o acesso a diferentes autores de diversas partes do mundo como Inglaterra, Espanha, Estados Unidos), os aplicativos para smartphone ( através do qual foi lido o livro Alice in Wonderland), os comandos de edição de texto, este artigo provavelmente não existiria.

Porém, como instrumento reflexivo, o texto procura instigar os designers para uma breve análise de onde estamos e para onde queremos ir, levando em consideração que este profissional é elemento fundamental na investigação e na proposição de novos produtos/serviços, e talvez principalmente na mediação e fomentação de novos valores que nos guiem a um estilo de vida sustentável. Thackara (2013) argumenta que "design for sustainability, it is turning out, is not about designers telling other people how to live. it's about the cocreation of tools enabling platforms that make it easier for people to share resources - such as energy, matter, time, skills, software, space or food ". (THACKARA apud WALKER, 2013, p. XXV)

E em tempos de digitalização da vida surgem novas abordagens tais como a cyber-sustentabilidade proposta por Knowles:

understanding of cyber-sustainability ought to include more than just the environmental impact of technologies for cyberspace. We should also be asking question about how well the Web satisfies our uniquely human needs in facilitating fulfilling social engagements (...) and in helping us flourish and achieve our human potential. Thus Web-based actives should enable the design intervention to cyber-sustainability. (KNOWLES apud WALKER, 2013, p.494)

Sendo assim podemos inferir que a digitalização apesar de se apresentar como uma possível solução de design para as questões relativas a sustentabilidade ambiental, apresenta-se ao mesmo tempo como um possível problema. Um retorno a um estágio pré-digital se mostra como inviável e inimaginável, e talvez o caminho seja buscar formas de humanização dentro dessa nova realidade, tentar alcançar o equilíbrio entre tecnológico e o humano. O designer como profissional multidisplinar e focado no usuário pode ser entendido como uma importante ferramenta nesse processo. Dessa maneira é fundamental buscarmos novas formas de atuação que permitam o desenvolvimento e aplicação desse potencial.

\section{REFERÊNCIAS}

BAUMAN, Zygmunt. A ética é possível num mundo de consumidores?. Rio de Janeiro: Zahar, 2011. 
CARROLL, Evan et. al. 2011. Your digital afterlife: when facebook, flirck and twitter are your estate, what's your legacy?. Berkeley: New Riders.

CARROLL, Lewis. Alice's adventures in wonderland. London: Macmillam, 1920. Disponível na internet por http em: <https://play.google.com/books/reader? id=Y7sOAAAAIAAJ\&printsec=frontcover\&output=reader\&authuser=0\&hl=pt_BR\&pg=G BS.PP13.w.0.1.0>. Acesso em 15 out. 2013

CHATFIELD, Tom. How to thrive in the digital age. London: Pan Macmillan, 2012.

EHRENFELD, John et. al. Flourishing: A Frank Conversation about Sustainability. California: Stanford University Press, 2013.

EHRENFELD, John. Sustainability by Design: A Subversive Strategy for Transforming Our Consumer Culture. USA: Yale University Press, 2008

FLINT, R. Warren et. al. Living a sustainable lifestyle for our children's future . Lincoln: iUniverse,2001.

FLUSSER, Vilém. O mundo codificado: por uma filosofia do design e da comunicação. Organização: Rafael Cardoso. Tradução: Raquel Abi-Sâmara. São Paulo: Cosac Naify, 2007.

FRIEDMAN, Thomas L. 0 mundo é plano: uma breve história do século XXI. Tradução de Cristiana Serra S. Duarte. Rio de Janeiro: Objetiva,2005.

FRY, Tony. 2011. Design as politics . New York: Berg.

HERCULANO, Selene. A qualidade de vida e seus indicadores in Qualidade de Vida e Riscos Ambientais. Niterói: Eduff, 2000.

JURIN, Richard. Principles of sustainable living: a new vision for health, happiness, and prosperity. USA,2012.

LÉVY, Pierre. Cibercultura. Tradução Carlos Irineu da Costa. São Paulo: Editora 34, 2 ed., 2007.

MANZINI, Ezio et. al. O desenvolvimento de produtos sustentáveis. São Paulo: Edusp, 2002.

MORIN,Edgar. A cabeça bem-feita: repensar a reform reformas o pensamento. Tradução Eloá Jacobina. Rio de Janeiro: Bertrand, 8 ed., 2003.

RUSHKOFF, Douglas . Program or be progammed: ten comands for a digital age. USA: Bookmobile,2010.

TAPIAS, José Antônio Pérez. Internautas e náufragos: a busca do sentido na cultura digital. São Paulo: Edições Loyola,2006.

VILCHES,Lorenzo. A migração digital. Tradução Maria Immacolata Vassalo de Lopes. São Paulo: Edições Loyola,2003.

WALKER, Stuart et. al. (org.) The Handbook of Design for Sustainability. Londres: Bloomsbury, 2013. 